\title{
Reduced Canonical Forms of Stoppers
}

\author{
Aaron N. Siegel \\ Mathematical Sciences Research Institute \\ 17 Gauss Way, Berkeley, CA 94720 \\ aaron.n.siegel@gmail.com
}

Submitted: May 12, 2006; Accepted: Jun 26, 2006; Published: Jul 28, 2006

Mathematics Subject Classification: 91A46

\begin{abstract}
The reduced canonical form of a loopfree game $G$ is the simplest game infinitesimally close to $G$. Reduced canonical forms were introduced by Calistrate, and Grossman and Siegel provided an alternate proof of their existence. In this paper, we show that the Grossman-Siegel construction generalizes to find reduced canonical forms of certain loopy games.
\end{abstract}

\section{Introduction}

The reduced canonical form $\bar{G}$ of a loopfree game $G$ is the simplest game infinitesimally close to $G$. Reduced canonical forms were introduced by Calistrate [4], who suggested a construction for $\bar{G}$. Calistrate's construction was recently proved correct by Grossman and Siegel [6], who also gave a second, quite different, construction of $\bar{G}$. In this paper we show that the Grossman-Siegel construction generalizes to a class of loopy games known as stoppers.

The arsenal of tools available in the study of loopy games is currently rather limited. The temperature theory and the theory of atomic weights, two of the most familiar techniques used to attack loopfree games, have not yet been adequately generalized to stoppers; though Berlekamp and others have extended the temperature theory to many loopy Go positions [2]. The reduced canonical form is therefore a welcome addition to the theory. It has already proven to be a useful tool in the study of loopfree games, particularly in situations where temperatures and atomic weights yield little information; see, for example, [7, Section 7].

In Section 2 we review the basic theory of stoppers and develop the necessary machinery for carrying out the reduction argument. Section 3 presents the construction. Finally, Section 4 poses some interesting open problems and directions for further research. 

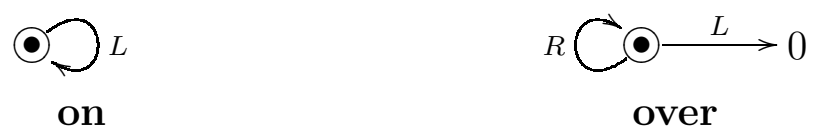

Figure 1: The graphs of on and over.

\section{Preliminaries}

By a loopy game $\gamma$ we mean a directed graph with separate Left and Right edge sets and an identified start vertex. If the play of $\gamma$ terminates, then the player who made the last move wins; otherwise the game is drawn. Throughout this paper we will assume that all loopy games are finite, and this assumption will be built in to all of our definitions.

The theory of loopy games was introduced in [5] and elaborated substantially in Winning Ways [3]. See [9] for an updated introduction. In this section we briefly review the definitions and facts that will be needed for this paper.

A path (walk) in the graph of $\gamma$ is alternating if its edges alternate in color. We say that the path (walk) is Left-alternating or Right-alternating if its first edge is a Left or Right edge, respectively.

Likewise, a cycle in the graph of $\gamma$ is alternating if it is even-length and its edges alternate in color. A game $\gamma$ is said to be a stopper if its graph contains no alternating cycles.

In many important respects, stoppers behave just like loopfree games. The following facts are established in [3].

Fact 2.1. Let $G$ be a stopper, and suppose $G^{\prime}$ is obtained from $G$ by eliminating a dominated option or bypassing a reversible one. Then $G^{\prime}$ is also a stopper and $G^{\prime}=G$.

Fact 2.2. Let $G$ and $H$ be stoppers with $G=H$. Suppose that neither $G$ nor $H$ has any dominated or reversible moves. Then for every $G^{L}$ there is an $H^{L}$ with $G^{L}=H^{L}$, and vice versa; likewise for Right options.

Two particular stoppers are critically important to the subsequent analysis: the games on $=\{\mathbf{o n} \mid\}$ and over $=\{0 \mid$ over $\}$. Their graphs are shown in Figure 1. We also define off $=-$ on $=\{\mid$ off $\}$ and under $=-$ over $=\{$ under $\mid 0\}$.

Note that when a stopper is played in isolation, play is guaranteed to terminate (since infinite play would necessarily traverse an alternating cycle). However, infinite play is still possible among sums of stoppers. For example, in the game on + off, both players may pass indefinitely.

\section{A Note on Induction}

Because stoppers have no alternating cycles, we can often induct along alternating sequences of play. The following definition formalizes this principle. 
Definition 2.3. Let $\gamma$ be a loopy game. The Left (Right) stopping distance of $\gamma$, denoted $\mathscr{D}^{L}(\gamma)\left(\mathscr{D}^{R}(\gamma)\right)$, is the length of the longest Left- (Right-) alternating walk proceeding from $\gamma$. We write $\mathscr{D}^{L}(\gamma)=\infty\left(\mathscr{D}^{R}(\gamma)=\infty\right)$ if no such maximum exists.

Clearly, if $G$ is a stopper, then $\mathscr{D}^{L}(G), \mathscr{D}^{R}(G)<\infty$. Since $\mathscr{D}^{R}\left(G^{L}\right)<\mathscr{D}^{L}(G)$ and $\mathscr{D}^{L}\left(G^{R}\right)<\mathscr{D}^{R}(G)$ for all $G^{L}, G^{R}$, we can use stopping distances as the basis for induction. This observation will simplify many subsequent arguments, though we will seldom refer explicitly to $\mathscr{D}^{L}(G), \mathscr{D}^{R}(G)$.

\section{Strategies}

Let $\gamma$ be a loopy game and let $\mathscr{A}$ be the set of all followers of $\gamma$. A Left strategy for $\gamma$ is a partial map $S: \mathscr{A} \rightarrow \mathscr{A}$ such that, whenever Left can move from some $\delta \in \mathscr{A}$, then $S(\delta)$ is defined and $S(\delta)=$ some $\delta^{L}$. A Left strategy $S$ is a second-player survival (winning) strategy for $\gamma$ if Left, playing second according to $S$, achieves at least a draw (win), no matter how Right plays. We say that Left can survive (win) $\gamma$ playing second if there exists a second-player Left survival (winning) strategy for $\gamma$.

Right strategies and first-player strategies are defined analogously.

Definition 2.4. Let $S$ be a Left strategy for $\gamma$. We say that $S$ is a complete survival strategy provided that, whenever Left can survive some follower $\delta \in \mathscr{A}$, then he can survive $\delta$ by playing according to $S$.

It is easy to see that every stopper $G$ admits a complete Left survival strategy: if $H$ is a follower of $G$, then $S(H)$ simply follows the recommendation of an arbitrary first-player Left survival strategy, whenever one exists. In fact, all loopy games admit complete survival strategies, but the general case is somewhat more complicated; see [10] for a proof.

We will need the following characterization of $\leq$, which is established in [3].

Fact 2.5. Let $G, H$ be stoppers. Then $G \leq H$ iff Left can survive $H-G$, playing second.

The following facts are easily verified using Fact 2.5.

Fact 2.6. Let $x>0$ be any positive number. Then $0<$ over $<x<$ on.

Fact 2.7. over + over $=$ over and on + on $=$ on .

Fact 2.8. If $G$ is any stopper, then $G \leq$ on.

We also have the following lemma.

Lemma 2.9 (Swivel Chair Lemma). Let $\gamma, \delta$ be arbitrary loopy games and let $G$ be a stopper. If Left can survive both $\gamma-G$ and $G-\delta$ playing second, then he can survive $\gamma-\delta$ playing second.

Note that Fact 2.5 requires the hypothesis that $G$ and $H$ are stoppers, so the Swivel Chair Lemma is not completely trivial. 
Proof of Lemma 2.9. Let $\alpha-\beta$ be a follower of $\gamma-\delta$. We say that $\alpha-\beta$ is safe if Left can survive both $\alpha-H$ and $H-\beta$ moving second, for some follower $H$ of $G$. By assumption, $\gamma-\delta$ is safe. Therefore, it suffices to show that if Right moves from any safe position, then Left can return to another safe position.

Let $\alpha-\beta$ be safe, and suppose without loss of generality that Right moves to $\alpha^{R}-\beta$. Since $\alpha-\beta$ is safe, there exists a follower $H$ of $G$ such that Left can survive $\alpha^{R}-H$ playing first and $H-\beta$ playing second. Choose such $H$ with $\mathscr{D}^{R}(H)$ minimal and let $S$ and $T$ be Left survival strategies for $\alpha^{R}-H$ and $H-\beta$, respectively.

Now if $S\left(\alpha^{R}-H\right)=\alpha^{R L}-H$, then $\alpha^{R L}-\beta$ is safe, and we are done. Otherwise, $S\left(\alpha^{R}-H\right)=\alpha^{R}-H^{R}$. Now consider $T\left(H^{R}-\beta\right)$. It cannot be the case that $T\left(H^{R}-\beta\right)=$ $T\left(H^{R L}-\beta\right)$ : for then Left could survive $\alpha^{R}-H^{R L}$ playing first, as well as $H^{R L}-\beta$ playing second; but $\mathscr{D}^{R}\left(H^{R L}\right)<\mathscr{D}^{R}(H)$, contradicting minimality of $\mathscr{D}^{R}(H)$. So in fact $T\left(H^{R}-\beta\right)=H^{R}-\beta^{R}$, whence $\alpha^{R}-\beta^{R}$ is safe.

\section{Infinitesimals and Stops}

An arbitrary game $\gamma$ is infinitesimal if $-x<\gamma<x$, for all numbers $x>0$. It is wellknown that a loopfree game $G$ is infinitesimal iff its Left and Right stops are both zero. We will need a suitable generalization of this fact.

Definition 2.10. Let $G$ be a stopper. $G$ is said to be a stop iff either $G$ is a number, $G=$ on, or $G=$ off. Likewise, we say that $G$ is stoppish iff either $G$ is numberish, $G=$ on, or $G=$ off.

We begin with some easy generalizations of well-known facts about loopfree games. As always, we write $G \triangleleft H$ to mean $G \geq H$ and $G \bowtie H$ to mean $G \not L H$.

Theorem 2.11 (Simplicity Theorem). Let $G$ be a stopper and suppose that, for some number $x$, we have $G^{L} \triangleleft x$ for all $G^{L}$ and $x \triangleleft G^{R}$ for all $G^{R}$. Then $G$ is a number.

Proof. Identical to the proof in the loopfree case.

Theorem 2.12 (Stop Avoidance Theorem). Let $G$ be a stopper and let $x$ be a stop. Assume that $G$ is not a stop and that Left, playing first, can survive $G+x$ by moving in $x$. Then he can also survive by moving in $G$.

Proof. Clearly $x \neq$ off, since Left has no moves in off. If $x=\mathbf{o n}$, then Left can survive $\alpha+x$ for any $\alpha$. Since $G$ is not a stop, Left must have a move in $G$, so he can survive $G^{L}+$ on and there is nothing more to prove.

Now assume that $x$ is a number. We proceed as in the loopfree case. Put $x_{0}=x$. Recursively on $n$, if $x_{n}$ has a Left option, put $x_{n+1}=x_{n}^{L}$. Since $x$ is a number, only finitely many $x_{n}$ 's can be so defined. Let $n$ be the largest integer such that Left can survive $G+x_{n}$ moving second. (Such an $n$ must exist, since by assumption Left can survive $G+x_{1}$ moving second.) Now if Right could also survive $G+x_{n}$ moving second, then we would have $G=-x_{n}$, contradicting the assumption that $G$ is not a stop. So Left 
must have a winning move from $G+x_{n}$. By definition of $n$, his move to $G+x_{n}^{L}$ (if it exists) is losing, so his winning move must be to $G^{L}+x_{n}$. But $x$ is a number, so $x>x_{n}$ and we are done.

Clearly the stops are totally-ordered, so the max and min of stops are well-defined. Thus we can make the following definition, by induction on stopping distance.

Definition 2.13. Let $G$ be a stopper. The Left and Right stops of $G$, denoted $L_{0}(G)$ and $R_{0}(G)$, are defined as follows.

$$
L_{0}(G)=\left\{\begin{array}{ll}
G & \text { if } G \text { is a stop; } \\
\max \left\{R_{0}\left(G^{L}\right)\right\} & \text { otherwise. }
\end{array} R_{0}(G)= \begin{cases}G & \text { if } G \text { is a stop; } \\
\min \left\{L_{0}\left(G^{R}\right)\right\} & \text { otherwise. }\end{cases}\right.
$$

Lemma 2.14. Let $G$ be a stopper and $x$ a number. If $L_{0}(G)>x$, then $G \mathbb{D} x$; if $R_{0}(G)<x$, then $G \triangleleft x$.

Proof. We prove the first assertion; the second follows by symmetry. Suppose that $L_{0}(G)>x$. If $G$ is a stop, then $G=L_{0}(G)$, so trivially $G>x$. Otherwise, let $G^{L}$ be such that $R_{0}\left(G^{L}\right)=L_{0}(G)$. Then $R_{0}\left(G^{L}\right)>x$. If $G^{L}$ is a stop, then $G^{L}>x$ and hence $G \bowtie x$. Otherwise, $\min \left\{L_{0}\left(G^{L R}\right)\right\}=R_{0}\left(G^{L}\right)>x$. We can assume (by induction on stopping distance) that $G^{L R} \bowtie x$ for each $G^{L R}$, so by the Stop Avoidance Theorem $G^{L} \geq x$. Thus $G \Vdash x$.

Lemma 2.15. $L_{0}(G) \geq R_{0}(G)$ for every stopper $G$.

Proof. It suffices to show that if

$$
\max \left\{R_{0}\left(G^{L}\right)\right\}<x<\min \left\{L_{0}\left(G^{R}\right)\right\}
$$

for some number $x$, then $G$ is itself a number. But by the previous lemma, $G^{L} \triangleleft x$ for each $G^{L}$ and $x \triangleleft G^{R}$ for each $G^{R}$, so the desired conclusion follows from the Simplicity Theorem.

Corollary 2.16. Let $G$ be a stopper. If $R_{0}(G)=\mathbf{o n}$, then $G=\mathbf{o n}$; if $L_{0}(G)=\mathbf{o f f}$, then $G=$ off.

Proof. We prove the first assertion; the proof of the second is identical. It suffices to show that $G$ is a stop, since then the conclusion follows by definition of $R_{0}$.

Assume (for contradiction) that $G$ is not a stop. Now $G \leq$ on (since this is true for any game). We will show that Left, playing second, can survive $G-\mathbf{o n}$, whence $G=\mathbf{o n}$, which is a stop.

It suffices to show that if Right moves from any position $X-\mathbf{o n}$, where $R_{0}(X)=\mathbf{o n}$, then Left can return to a position of the same form. If $X$ is a stop, then $X=$ on and this is immediate. If Right moves to some $X^{R}-\mathbf{o n}$, then $L_{0}\left(X^{R}\right)=\mathbf{o n}$. If $X^{R}$ is a stop, then $X^{R}=$ on and we are done. Otherwise, there is some $X^{R L}$ with $R_{0}\left(X^{R L}\right)=\mathbf{o n}$, and Left can move to $X^{R L}-$ on.

Finally, if Right passes from $X$ - on, then by the previous lemma we have $L_{0}(X) \geq$ $R_{0}(X)=\mathbf{o n}$, whence $L_{0}(X)=\mathbf{o n}$. So there is some $X^{L}$ with $R_{0}\left(X^{L}\right)=\mathbf{o n}$, and Left can move to $X^{L}-$ on. 


\section{Reduction}

This section describes the construction of $\bar{G}$. The following definition is fundamental.

Definition 3.1. Let $G, H$ be any two games.

(a) $G \geq \operatorname{Inf} H$ iff $G+x \geq H$ for all numbers $x>0$.

(b) $G \equiv_{\operatorname{Inf}} H$ iff $G \geq_{\operatorname{Inf}} H$ and $H \geq_{\operatorname{Inf}} G$.

Notice that if $G$ is a stopper and $x$ is a number in simplest form, then since $x$ is loopfree, $G+x$ is also a stopper. We can therefore evaluate the condition $G+x \geq H$ in (a) using Fact 2.5.

Lemma 3.2. $\geq_{\operatorname{Inf}}$ is transitive.

Proof. Suppose $G \geq_{\operatorname{Inf}} H$ and $H \geq \operatorname{Inf} K$, and fix $x>0$. Then $G+\frac{x}{2} \geq H$ and $H+\frac{x}{2} \geq K$, so $G+x \geq K$, as needed.

In [6] we defined Inf-dominated and Inf-reversible options of loopfree games in terms of $\geq_{\text {Inf }}$. We then showed that if $G$ is not numberish, and Inf-dominated options are eliminated from $G$ or Inf-reversible ones bypassed, then the value of $G$ is perturbed at most by an infinitesimal. We will now extend this technique to stoppers. The main complication is that we can no longer proceed by induction: because $G$ might be loopy, it is not safe to assume that its followers are all in reduced canonical form.

We begin with some necessary spadework.

Lemma 3.3. Let $G$ be a stopper and let $x$ be a number. If $L_{0}(G)<x$, then Left, playing second, can win $x-G$.

Proof. Since $x-G$ is a stopper, it suffices to show that Left can survive $x-G$. Now if $G$ is a stop, then there is nothing to prove. Otherwise, by the Stop Avoidance Theorem, we may assume Right moves to $x-G^{L}$. If $G^{L}$ is a stop, then $G^{L} \leq L_{0}(G)<x$, and we are done. Finally, if $G^{L}$ is not a stop, then there is some $G^{L R}$ such that

$$
L_{0}\left(G^{L R}\right)=R_{0}\left(G^{L}\right) \leq L_{0}(G)<x
$$

so Left can respond to $x-G^{L R}$.

Lemma 3.4. Let $G, H$ be stoppers.

(a) If $R_{0}(G) \geq L_{0}(H)$, then $G \geq_{\operatorname{Inf}} H$.

(b) If $G \geq \operatorname{Inf} H$, then $L_{0}(G) \geq L_{0}(H)$ and $R_{0}(G) \geq R_{0}(H)$. 
Proof. (a) Fix $x>0$; we will show that Left, playing second, can survive $G-H+x$. It suffices to show that if Right moves from a position of the form

$$
X-Y+z, \text { with } R_{0}(X) \geq L_{0}(Y) \text { and } z>0,
$$

then Left can either survive outright, or respond to a position of the same form.

If $X$ and $Y$ are both stops, then necessarily $X \geq Y$, and the conclusion is immediate. Otherwise, we may assume by symmetry that $X$ is not a stop. There are three cases.

Case 1: If Right moves to $X-Y+z^{R}$, then since $L_{0}(X) \geq R_{0}(X)$, there is some $X^{L}$ with $R_{0}\left(X^{L}\right) \geq R_{0}(X) \geq L_{0}(Y)$. Since $z^{R}>z>0$, it suffices for Left to move to $X^{L}-Y+z^{R}$.

Case 2: Suppose instead that Right moves to $X^{R}-Y+z$. If $X^{R}$ is not a stop, then for some $X^{R L}$, we have $R_{0}\left(X^{R L}\right)=L_{0}\left(X^{R}\right) \geq R_{0}(X)$, whence $X^{R L}-Y+x$ has the desired form. If $X^{R}$ is a stop but $Y$ is not, then for some $Y^{R}$ we have $L_{0}\left(Y^{R}\right)=R_{0}(Y) \leq L_{0}(Y)$, and $X^{R}-Y^{R}+z$ has the desired form. Finally, if $X^{R}$ and $Y$ are both stops, then $X^{R} \geq Y$. Since $z>0$, there must be some $z^{L} \geq 0$, and Left survives by moving to $X^{R}-Y+z^{L}$.

Case 3: If Right moves to $X-Y^{L}+z$, then the argument is like Case 1 (if $Y$ is a stop) or Case 2 (if $Y$ is not a stop).

(b) We show that $L_{0}(G) \geq L_{0}(H)$; the proof that $R_{0}(G) \geq R_{0}(H)$ is similar. Arguing by contrapositive, suppose that $L_{0}(G)<L_{0}(H)$. If $L_{0}(G)=$ off, then by Corollary 2.16 $G=$ off. Since $L_{0}(H)>$ off, part (a) of this lemma implies that $H>n$ for some integer $n$. Thus $G \Varangle_{\text {Inf } H} H$. The same argument applies if $L_{0}(H)=$ on.

Now suppose $L_{0}(G)$ and $L_{0}(H)$ are both numbers. Put

$$
x=\frac{L_{0}(H)-L_{0}(G)}{2} .
$$

To complete the proof, we will show that $G+x \triangleleft H$. If $H$ is a stop, then the conclusion is a consequence of Lemma 3.3. If $H$ is not a stop, then we describe a first-player Right winning strategy for $G-H+x$. Let $H^{L}$ be such that $R_{0}\left(H^{L}\right)=L_{0}(H)$. Now $L_{0}(G)+x<R_{0}\left(H^{L}\right)$. We conclude by showing the following: if Left moves from any position of the form

$$
X-Y+z, \text { with } L_{0}(X)+z<R_{0}(Y)
$$

then Right has a response that either wins outright, or returns to another position of the form $(\dagger)$. Furthermore, Right's response will occur in the same component $(X+z)$ or $(-Y)$ as Left's move. Since both are stoppers, this shows that play must terminate.

Suppose first that Left moves to $X^{L}-Y+z$. Then $R_{0}\left(X^{L}\right) \leq L_{0}(X)$. If $X^{L}$ and $Y$ are both stops, there is nothing more to prove. If $X^{L}$ is a stop but $Y$ is not, then Right moves to $X^{L}-Y^{L}+z$ with $R_{0}\left(Y^{L}\right)=L_{0}(Y) \geq R_{0}(Y)$; by Lemma 3.3 this is a winning move. If $X^{L}$ is not a stop, then Right moves to $X^{L R}-Y+z$ with $L_{0}\left(X^{L R}\right)=R_{0}\left(X^{L}\right) \leq L_{0}(X)$. A similar argument applies if Left moves from $(\dagger)$ to $X-Y^{R}+z$.

Finally, suppose Left moves to $X-Y+z^{L}$. If either $X$ or $Y$ is a stop, then by Lemma 3.3 Right has a winning move in the other component. Otherwise, there is some $X^{R}$ with $L_{0}\left(X^{R}\right)=R_{0}(X) \leq L_{0}(X)$. Since $z^{L}<z$, the position $X^{R}-Y+z^{L}$ has the form $(\dagger)$. 
Corollary 3.5. Let $G$ be a stopper. Then $G$ is stoppish iff $L_{0}(G)=R_{0}(G)$.

Proof. The forward direction is trivial. For the converse, suppose $L_{0}(G)=R_{0}(G)=x$. If $x=\mathbf{o n}$, then by Corollary $2.16 G=\mathbf{o n}$; likewise if $x=\mathbf{o f f}$. So assume $x$ is a number. Then $R_{0}(G)=L_{0}(x)$, so by Lemma 3.4(a), we have $G \geq_{\operatorname{Inf}} x$. Similarly, $R_{0}(x)=L_{0}(G)$, so $x \geq \operatorname{Inf} G$, and we conclude that $G$ is $x$-ish.

Lemma 3.6. The following are equivalent:

(i) $G \geq \operatorname{Inf} H$;

(ii) Left, playing second, can survive $G-H+$ over.

Proof. (i) $\Rightarrow$ (ii): Arguing by contrapositive, suppose that Right has a winning move from $G-H+$ over and let $S$ be her first-player winning strategy. Now if $G-H+$ over is played according to $S$, then since $S$ guarantees that play will be finite, we know that Right will never have to move twice from the same position. Let $n$ be the total number of followers of $G-H$. We have shown that, if Right plays according to $S$, then she will make at most $n$ moves before reaching a position from which Left has no play. Therefore $S$ suffices as a winning strategy for

$$
G-H+\frac{1}{2^{n+1}}
$$

whence $H \mathbb{} \triangleright G+2^{-(n+1)}$.

(ii) $\Rightarrow$ (i): If $x>0$ is a number, then $x>$ over, so Left can survive $x$ - over. By the Swivel Chair Lemma, he can survive $G-H+x$.

Most of the proofs that follow involve exhibiting a Left survival strategy $S^{\prime}$ for some position $G-G^{\prime}+$ over. Usually $G^{\prime}$ will be a modified copy of $G$ (for example, with an Infdominated option removed from some follower), and $S^{\prime}$ will be derived from a complete survival strategy $S$ for $G-G+$ over. It will be essential that $S^{\prime}$ preserves the component over until a point is reached where Left absolutely must remove it. The following lemma is key.

Lemma 3.7 (over Avoidance Theorem). Let $G$ be a stopper and let $\alpha$ be an arbitrary loopy game. Assume that $G$ is not a stop, and suppose that Left, playing first, can survive

$$
G+\alpha+\text { over }
$$

by moving from over to 0 . Then he can also survive by moving in $G$.

Proof. Let $G^{L}$ be any Left option with $R_{0}\left(G^{L}\right)=L_{0}(G)$. By Lemmas 3.4(a) and 3.6, we know that Left, playing second, can survive $G^{L}-G+$ over.

Now by the hypotheses of the lemma, Left (playing second) can also survive $G+\alpha$. Since $G$ is a stopper, the Swivel Chair Lemma establishes that he can survive $G^{L}+\alpha+$ over. 
Definition 3.8. A stopper $G$ is partially reduced if every stoppish follower of $G$ is a stop in canonical form.

Lemma 3.9 (Replacement Lemma). Let $G$ be any stopper and let $G^{\prime}$ be the game obtained by replacing every stoppish follower $K$ of $G$ with the associated stop $L_{0}(K)$ (in canonical form). Then $G^{\prime}$ is partially reduced and $G \equiv_{\operatorname{Inf}} G^{\prime}$.

Proof. To see that $G^{\prime}$ is partially reduced, it suffices to show that $L_{0}(K)=L_{0}\left(K^{\prime}\right)$ and $R_{0}(K)=R_{0}\left(K^{\prime}\right)$ for every follower $K$ of $G$. If $K$ is a stop, then this is immediate. Otherwise, to show that $L_{0}(K)=L_{0}\left(K^{\prime}\right)$, we may assume (by induction on stopping distance) that $R_{0}\left(K^{L}\right)=R_{0}\left(\left(K^{L}\right)^{\prime}\right)$ for every Left option $K^{L}$; the conclusion is then trivial. The same argument shows that $R_{0}(K)=R_{0}\left(K^{\prime}\right)$.

We now show that $G \equiv_{\operatorname{Inf}} G^{\prime}$. By Lemma 3.6, it suffices to show that Left, playing second, can survive both $G-G^{\prime}+$ over and $G^{\prime}-G+$ over. We will give the proof for $G-G^{\prime}+$ over; the other case is identical.

Let $S$ be a complete survival strategy for $G-G+$ over. By the over Avoidance Theorem, we may assume that $S$ recommends a move in over only when both of the other components are stops.

Now Left plays $G-G^{\prime}+$ over according to $S$ until the $-G^{\prime}$ component reaches a stop. Let $X-Y^{\prime}+$ over be the resulting position, and let $X-Y+$ over be the corresponding subposition of $G-G+$ over, so that $Y^{\prime}$ is a stop and $Y$ is $Y^{\prime}$-ish. Consider $S(X-Y+$ over $)$. If $S(X-Y+$ over $)=X^{L}-Y+$ over, then $X^{L} \geq \operatorname{Inf} Y$, so by Lemma 3.4(b), we have $R_{0}\left(X^{L}\right) \geq R_{0}(Y)=Y^{\prime}$. Therefore Left can survive $X^{L}-Y^{\prime}+$ over by playing optimally in $X^{L}$ until a stop is reached, and then moving in over.

If $S(X-Y+$ over $)=X-Y^{R}+$ over, then $L_{0}(X) \geq L_{0}\left(Y^{R}\right) \geq R_{0}(Y)=Y^{\prime}$. So Left can survive $X-Y^{\prime}+$ over by playing optimally in $X$ until a stop is reached, and then moving in over.

Next we define the critical notions of Inf-dominated and Inf-reversible options.

Definition 3.10. Let $G$ be a partially reduced stopper and let $G^{L}$ be a Left option of $G$.

(a) $G^{L}$ is said to be Inf-dominated if $G^{L^{\prime}} \geq \operatorname{Inf} G^{L}$ for some other Left option $G^{L^{\prime}}$.

(b) $G^{L}$ is said to be Inf-reversible if $G^{L R} \leq_{\text {Inf }} G$ for some Right option $G^{L R}$.

Inf-dominated and Inf-reversible Right options are defined analogously.

Lemma 3.11. Let $G$ be a partially reduced stopper and let $H$ be any follower of $G$. Suppose $H^{L}$ is Inf-dominated by $H^{L^{\prime}}$ and let $G^{\prime}$ be obtained from $G$ by eliminating $H \rightarrow$ $H^{L}$. Then $G^{\prime}$ is partially reduced and $G^{\prime} \equiv_{\operatorname{Inf}} G$.

Proof. To see that $G^{\prime}$ is partially reduced, it suffices to show that $L_{0}(K)=L_{0}\left(K^{\prime}\right)$ and $R_{0}(K)=R_{0}\left(K^{\prime}\right)$ for every follower $K$ of $G$. This is a simple induction on the stopping distance of $K$; the only complication occurs when $K=H$. In that case, $H^{L^{\prime}} \geq_{\operatorname{Inf}} H^{L}$, so by Lemma 3.4(b) we have $R_{0}\left(H^{L^{\prime}}\right) \geq R_{0}\left(H^{L}\right)$. Therefore $H^{L}$ does not contribute to $L_{0}(H)$, and so $L_{0}\left(H^{\prime}\right)=L_{0}(H)$. 
Now it is immediate that $G^{\prime} \leq_{\operatorname{Inf}} G$. To complete the proof, we will show that Left, playing second, can survive $G^{\prime}-G+$ over.

Let $S$ be a complete Left survival strategy for $G-G+$ over. By the over Avoidance Theorem, we may assume that $S$ recommends a move in over only when the other components are stops. Define a Left strategy $S^{\prime}$ for $G^{\prime}-G+$ over as follows. $S^{\prime}$ is identical to $S$ except when $S$ recommends a move from $H-Y+$ over to $H^{L}-Y+$ over. In that case, $S^{\prime}$ recommends a move to $\left(H^{L^{\prime}}\right)^{\prime}-Y+$ over instead.

We claim that $S^{\prime}$ is a second-player survival strategy. We first show that, whenever Left (playing according to $S^{\prime}$ ) moves to a position $X^{\prime}-Y+$ over of $G^{\prime}-G+$ over, then he can survive the corresponding position $X-Y+$ over. Since $S^{\prime}$ is derived from $S$, this is automatic except when Left moves from $H^{\prime}-Y+$ over and $S$ recommends a move to $H^{L}-Y+$ over. But in that case, we know $H^{L} \geq_{\operatorname{Inf}} Y$ (since $S$ is a survival strategy), so $H^{L^{\prime}} \geq_{\text {Inf }} H^{L} \geq_{\text {Inf }} Y$. So in fact Left can survive $H^{L^{\prime}}-Y+$ over.

To complete the proof, we must show that if Left has a survival move and $S^{\prime}\left(X^{\prime}-\right.$ $Y+$ over $)=X^{\prime}-Y$, then $X^{\prime} \geq Y$. But by assumption on $S$, this will not happen unless $X$ is a stop. Therefore $H$ cannot be a follower of $X$ (since $G$ is partially reduced), and we conclude that $X=X^{\prime}$. But since $S$ is a survival strategy, we know that $X \geq Y$.

Lemma 3.12. Let $G$ be a partially reduced stopper and let $H$ be any follower of $G$. Suppose $H^{L_{1}}$ is Inf-reversible through $H^{L_{1} R_{1}}$ and let $G^{\prime}$ be obtained from $G$ by bypassing $H \rightarrow H^{L_{1}} \rightarrow H^{L_{1} R_{1}}$. Then $G^{\prime}$ is partially reduced and $G^{\prime} \equiv_{\operatorname{Inf}} G$.

Proof. As before, to see that $G^{\prime}$ is partially reduced, it suffices to show that $L_{0}(K)=$ $L_{0}\left(K^{\prime}\right)$ and $R_{0}(K)=R_{0}\left(K^{\prime}\right)$ for every follower $K$ of $G$. This is a simple induction on the stopping distance of $K$, except when $K=H$. In that case, we may assume that $R_{0}\left(H^{L}\right)=R_{0}\left(\left(H^{L}\right)^{\prime}\right)$ for every $H^{L}$, and we must show that $L_{0}(H)=L_{0}\left(H^{\prime}\right)$. There are two cases.

Case 1: $R_{0}\left(H^{L_{1}}\right)<L_{0}(H)$. Then $H^{L_{1}}$ does not contribute to $L_{0}(H)$, and the conclusion is trivial.

Case 2: $R_{0}\left(H^{L_{1}}\right)=L_{0}(H)$. Then $L_{0}\left(H^{L_{1} R_{1}}\right) \geq R_{0}\left(H^{L_{1}}\right)=L_{0}(H)$, so there is some $H^{L_{1} R_{1} L}$ with $R_{0}\left(H^{L_{1} R_{1} L}\right) \geq L_{0}(H)$. By induction, we may assume that $R_{0}\left(\left(H^{L_{1} R_{1} L}\right)^{\prime}\right)=$ $R_{0}\left(H^{L_{1} R_{1} L}\right)$. This shows that $L_{0}\left(H^{\prime}\right) \geq L_{0}(H)$.

However, we also know that $H^{L_{1} R_{1}} \leq_{\text {Inf }} H$, so by Lemma 3.4(b) we have $L_{0}\left(H^{L_{1} R_{1}}\right) \leq$ $L_{0}(H)$. Therefore every $H^{L_{1} R_{1} L}$ satisfies $R_{0}\left(H^{L_{1} R_{1} L}\right) \leq L_{0}(H)$, and by induction we conclude that $L_{0}\left(H^{\prime}\right) \leq L_{0}(H)$. Therefore $L_{0}\left(H^{\prime}\right)=L_{0}(H)$, as needed.

Next, we must show that Left, playing second, can survive both $G-G^{\prime}+$ over and $G^{\prime}-G+$ over. Let $S$ be a complete Left survival strategy for $G-G+$ over. By the over Avoidance Theorem, we may assume that $S$ recommends a move in over only in the case where the other components are stops.

First consider $G-G^{\prime}+$ over. Suppose Left can survive $X-H+$ over playing second. Then $X \geq_{\text {Inf }} H \geq_{\text {Inf }} H^{L_{1} R_{1}}$, so in fact Left can survive $X-H^{L_{1} R_{1}}+$ over playing second. Therefore, if Left can survive $X-H+$ over playing second, then he has a survival move from $X-H^{L_{1} R_{1} L}+$ over, for every $H^{L_{1} R_{1} L}$. It follows that $S$ suffices, without modification, as a survival strategy for $G-G^{\prime}+$ over. 
To complete the proof, we must show that Left, playing second, can survive $G^{\prime}-G+$ over. We will define a new strategy $S^{\prime}$ for $G^{\prime}-G+$ over.

$S^{\prime}$ is identical to $S$, except at those positions $\alpha=H-K+$ over such that Left has a survival move from $\alpha$, and $S(\alpha)=H^{L_{1}}-K+$ over. In that case, since $S$ is a complete survival strategy, Left must have a survival move from $\beta=H^{L_{1} R_{1}}-K+$ over. There are three cases; in each case, we specify the definition of $S^{\prime}\left(\alpha^{\prime}\right)$, where $\alpha^{\prime}=H^{\prime}-K+$ over.

Case 1: $S(\beta)=H^{L_{1} R_{1} L}-K+$ over. Then put $S^{\prime}\left(\alpha^{\prime}\right)=\left(H^{L_{1} R_{1} L}\right)^{\prime}-K+$ over.

Case 2: $S(\beta)=H^{L_{1} R_{1}}-K^{L}+$ over. Then put $S^{\prime}\left(\alpha^{\prime}\right)=H^{\prime}-K^{L}+$ over.

Case 3: $S(\beta)=H^{L_{1} R_{1}}-K$. Then by assumption on $S, H^{L_{1} R_{1}}$ and $K$ are both stops. Since $G$ is partially reduced and $H$ is not a stop, we know that $H$ is not stoppish. By Corollary 3.5, it follows that $L_{0}(H)>R_{0}(H)$. But $H^{L_{1} R_{1}} \leq_{\text {Inf }} H$, so Lemma 3.4(b) implies that $R_{0}\left(H^{L_{1} R_{1}}\right) \leq R_{0}(H)$. Combining these inequalities with the fact that $H^{L_{1} R_{1}}$ is a stop, we obtain

$$
L_{0}(H)>R_{0}(H) \geq R_{0}\left(H^{L_{1} R_{1}}\right)=H^{L_{1} R_{1}} .
$$

But $H^{L_{1} R_{1}} \geq R_{0}\left(H^{L_{1}}\right)$, so $L_{0}(H)>R_{0}\left(H^{L_{1}}\right)$, and we conclude that $H$ has some other Left option $H^{L^{\prime}} \neq H^{L_{1}}$ with $R_{0}\left(H^{L^{\prime}}\right)>H^{L_{1} R_{1}}$. Put $S^{\prime}\left(\alpha^{\prime}\right)=\left(H^{L^{\prime}}\right)^{\prime}-K+$ over.

This defines $S^{\prime}$. We now claim that $S^{\prime}$ is a survival strategy. We first show that if Right moves from any position of the form

$$
X^{\prime}-Y+\text { over, such that Left can survive } X-Y+\text { over, }
$$

then $S^{\prime}\left(\left(X^{\prime}-Y+\text { over }\right)^{R}\right)$ is another position of the form $(\dagger)$. This is clear except when $\left(X^{\prime}-Y+\text { over }\right)^{R}=H^{\prime}-K+$ over for some $K$, and $S(H-K+$ over $)=H^{L_{1}}-K+$ over. Then the proof depends on case. In Case 1, the conclusion is immediate. In Case 2, we have that $H \geq_{\text {Inf }} H^{L_{1} R_{1}} \geq_{\text {Inf }} K^{L}$, so in fact Left can survive $H-K^{L}+$ over. Finally, in Case 3, we have that $H^{L^{\prime}} \geq \operatorname{Inf} H^{L_{1} R_{1}}$ (Lemma 3.4(a)). Since $H^{L_{1} R_{1}} \geq K$, we conclude that Left can survive $H^{L^{\prime}}-K+$ over, as needed.

This completes the proof, except for the case where $S^{\prime}\left(X^{\prime}-Y+\right.$ over $)=X^{\prime}-Y$ (removing the over component). But then, by assumption on $S$, we know that $X$ and $Y$ are both stops. Since $H$ is stoppish and $G$ is partially reduced, $H$ is not a follower of $X$. We conclude that $X^{\prime}=X$, and since $S$ is a survival strategy, $X \geq Y$.

Definition 3.13. A stopper $G$ is said to be in reduced canonical form if $G$ is partially reduced and no follower of $G$ contains any Inf-dominated or Inf-reversible options.

Lemma 3.14. For any stopper $G$, there exists a stopper $G^{\prime}$ in reduced canonical form with $G^{\prime} \equiv_{\operatorname{Inf}} G$.

Proof. By the Replacement Lemma we can assume that $G$ is partially reduced. The conclusion then follows by repeated application of Lemmas 3.11 and 3.12.

We conclude by proving the uniqueness of the $G^{\prime}$ guaranteed by Lemma 3.14.

Theorem 3.15. Suppose $G \equiv_{\operatorname{Inf}} H$ and $G, H$ are in reduced canonical form. Then $G=H$. 
Proof. We will show that Left, playing second, can survive $G-H$. It suffices to show that if Right moves from a subposition $X-Y$ with $X \equiv_{\operatorname{Inf}} Y$, then Left can respond to another position that satisfies the same equivalence.

If $X$ and $Y$ are both stops, then $X=Y$, and the conclusion is trivial. Otherwise, neither is a stop, since this would imply that the other is stoppish, contradicting the fact that both are partially reduced. Suppose Right moves to $X^{R}-Y$ (the argument is the same if he moves to $\left.X-Y^{L}\right)$. Now $X \equiv_{\operatorname{Inf}} Y$, so Left has a survival move from $X^{R}-Y+$ over. Since $Y$ is not a number, the over Avoidance Theorem implies that Left has a survival move in either $X^{R}$ or $-Y$. It cannot be to $X^{R L}-Y+$ over, for this would imply $X^{R L} \geq_{\operatorname{Inf}} Y \equiv_{\operatorname{Inf}} X$, contradicting the fact that $X$ has no Inf-reversible moves. So it must be to $X^{R}-Y^{R}+$ over. Thus $X^{R} \geq \operatorname{Inf} Y^{R}$.

But Left also has a survival move from $Y^{R}-X+$ over, and by the same argument it must be to some $Y^{R}-X^{R^{\prime}}+$ over, so that

$$
X^{R} \geq_{\operatorname{Inf}} Y^{R} \geq_{\operatorname{Inf}} X^{R^{\prime}}
$$

Since $X$ has no Inf-dominated options, this implies $X^{R} \equiv_{\operatorname{Inf}} Y^{R} \equiv_{\operatorname{Inf}} X^{R^{\prime}}$, and Left can respond in $X^{R}-Y$ by moving to $X^{R}-Y^{R}$.

As a trivial consequence of these results, we have the following neat little theorem. Recall that if $G$ is a stopper, then a pass move is a move from $G$ to $G$. A stopper is a ripe plumtree if its only loops are pass moves (i.e., if its graph contains no $n$-cycles for any $n>1)$.

Theorem 3.16. Let $G$ be a stopper in reduced canonical form. Then $G$ contains no pass moves except from subpositions equal to on or off. In particular, if $G$ is any ripe plumtree and no subposition of $G$ is equal to on or off, then $G$ is infinitesimally close to a loopfree game.

Proof. Suppose (for contradiction) that $H^{R}=H$ for some follower $H$ of $G$. Since $H \neq$ off, the over Avoidance Theorem implies that Left can survive $H^{L}-H+$ over for some $H^{L}$. Therefore $H^{L} \geq_{\operatorname{Inf}} H$. But then Right's pass move is Inf-reversible through $H^{L}=H^{R L}$.

Note that Theorem 3.16 only applies to plumtrees. There certainly exist more complicated stoppers with no on or off positions that are not infinitesimally close to any loopfree game. Figure 2 illustrates a typical example.

\section{Generalizations and Open Problems}

Let $\mathcal{K}$ be a subgroup of $\mathcal{G}$, the group of finite loopfree games, and define $G \equiv_{\mathcal{K}} H$ iff $G-H \in \mathcal{K}$. This generalizes $\equiv_{\text {Inf }}$, which is equivalence modulo the group Inf of infinitesimals.

In [6] we defined a reduction modulo $\mathcal{K}$ to be a map $\rho: \mathcal{G} \rightarrow \mathcal{G}$ such that $\rho(G) \equiv_{\mathcal{K}} G$ for all $G ; \rho(x)=x$ for all numbers $x$; and $\rho(G)=\rho(H)$ whenever $G \equiv_{\mathcal{K}} H$. We observed 


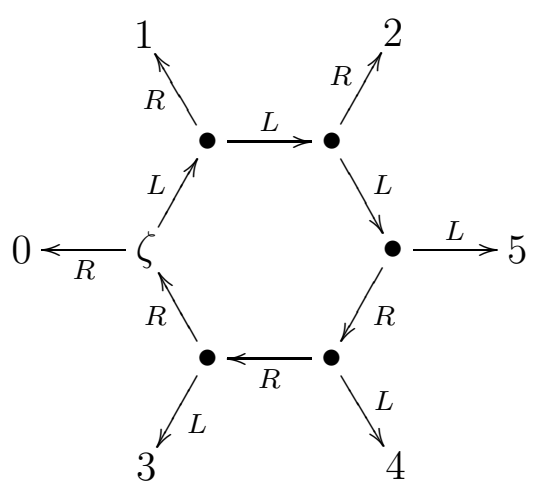

Figure 2: A stopper $\zeta$ that is not infinitesimally close to any loopfree game.

that the mapping $G \mapsto \bar{G}$ is a reduction modulo Inf, and asked whether one can exhibit any reductions modulo

$$
\operatorname{Inf}^{n}=\left\{G \in \mathcal{G}: k \cdot \downarrow_{n}<G<k \cdot \uparrow^{n} \text { for some } k\right\},
$$

or some other interesting subgroup of $\mathcal{G}$.

Stoppers can provide useful insights into this question. Suppose we expand our definition of reduction to allow mappings $\rho: \mathcal{G} \rightarrow \mathcal{S}$, where $\mathcal{S}$ is the set of stoppers, and drop the condition that $\rho$ preserve numbers. Then it is not hard to see that

$$
G \mapsto G+\text { over }
$$

is a reduction; for if $\varepsilon$ is a loopfree infinitesimal, then over $+\varepsilon=$ over. Unlike the examples considered in [6], it is surprisingly easy to define $G \mapsto G+$ over and prove that it is a reduction.

Now let $I$ be any stopper satisfying $I+I=I$. Then it is easy to see that

$$
\mathcal{K}_{I}=\{G \in \mathcal{G}: I+G=I\}
$$

is a subgroup of $\mathcal{G}$. For example, $\mathcal{K}_{\text {over }}=\operatorname{Inf}$, and $\mathcal{K}_{\text {on }}=\mathcal{G}$. Furthermore, the mapping $G \mapsto G+I$ is a reduction modulo $\mathcal{K}_{I}$. This mapping directly generalizes the reduction $G \mapsto G+$ over modulo Inf.

The importance of this observation stems from the fact that $\mathcal{S}$ contains a great many idempotents. Several of these are listed in Figure 8 of [10]. A typical example is

$$
\mathscr{I}_{n}=\left\{0|| 0, \operatorname{pass} \mid 0, \downarrow_{\rightarrow(n-2)} *\right\} \quad(n \geq 2),
$$

which satisfies $\uparrow^{n} \in \mathcal{K}_{\mathscr{I}_{n}}$, but $\uparrow^{n-1} \notin \mathcal{K}_{\mathscr{I}_{n}}$. This does not quite answer our question regarding reduction modulo $\operatorname{Inf}^{n}$ : we would prefer to exhibit a mapping $\mathcal{G} \rightarrow \mathcal{G}$. But perhaps a study of the action $G \mapsto G+\mathscr{I}_{n}$ might yield some insight.

Open Problem. Exhibit a reduction $\rho: \mathcal{G} \rightarrow \mathcal{G}$ modulo $\operatorname{Inf}^{2}$ (or some other "interesting" subgroup of $\mathcal{G}$ ). 
Finally, note that under + over is not a stopper, so the map $G \mapsto G+$ over does not define a mapping $\mathcal{S} \rightarrow \mathcal{S}$. However, it is true that $\alpha+$ under + over $\equiv_{\text {Inf }} \alpha$, for all loopy games $\alpha$. Furthermore, if $\alpha \equiv_{\operatorname{Inf}} \beta$, then $\alpha+$ under + over $=\beta+$ under + over. (Here $\equiv_{\text {Inf }}$ is defined just as for stoppers.) Therefore the mapping $\alpha \mapsto \alpha+$ under + over can be considered a reduction on the monoid $\mathcal{L}$ of all (finite) loopy games. This generalizes to the mapping $\alpha \mapsto \alpha+I-I$, for any idempotent $I$.

Many years ago, Clive Bach observed that it is difficult (if not impossible) to define the canonical form of an arbitrary loopy game. Perhaps one might have more success defining the reduced canonical form. For example, let $\kappa$ be Bach's Carousel [3]. As noted in Winning Ways, $\kappa^{+}$is not equivalent to a stopper. However, it is not hard to show that $\kappa^{+} \equiv_{\operatorname{Inf}}\{1 \mid 0\}$, in the usual sense:

$$
\{1 \mid 0\}+\text { under } \leq \kappa^{+} \leq\{1 \mid 0\}+\text { over }
$$

In Winning Ways, the authors asked: "Is there an alternative notion of simplest form that applies to all finite loopy games?" We conclude with the following specialization:

Question. Does the notion of reduced canonical form apply to all finite loopy games?

\section{References}

[1] M. Albert and R. J. Nowakowski, editors. Games of No Chance 3. MSRI Publications. Cambridge University Press, Cambridge, forthcoming.

[2] E. R. Berlekamp. The economist's view of combinatorial games. In Nowakowski [8], pages 365-405.

[3] E. R. Berlekamp, J. H. Conway, and R. K. Guy. Winning Ways for Your Mathematical Plays. A. K. Peters, Ltd., Natick, MA, second edition, 2001.

[4] D. Calistrate. The reduced canonical form of a game. In Nowakowski [8], pages 409-416.

[5] J. H. Conway. Loopy games. In B. Bollobás, editor, Advances in Graph Theory, number 3 in Ann. Discrete Math., pages 55-74, 1978.

[6] J. P. Grossman and A. N. Siegel. Reductions of partizan games. In Albert and Nowakowski [1].

[7] G. A. Mesdal. Partizan splittles. In Albert and Nowakowski [1].

[8] R. J. Nowakowski, editor. Games of No Chance. Number 29 in MSRI Publications. Cambridge University Press, Cambridge, 1996.

[9] A. N. Siegel. Coping with cycles. In Albert and Nowakowski [1].

[10] A. N. Siegel. New results in loopy games. In Albert and Nowakowski [1]. 